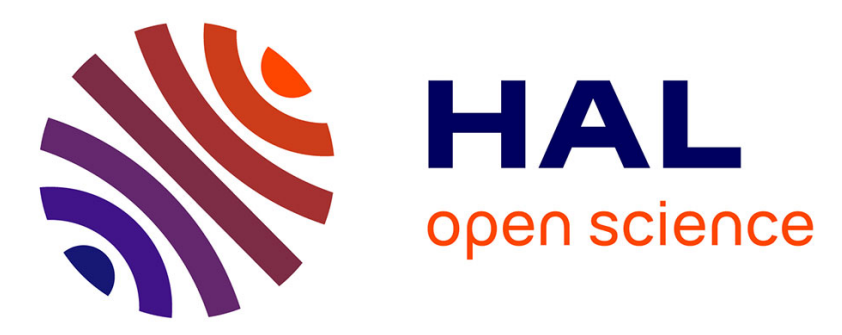

\title{
Stability of parallel wake flows in quasigeostrophic and frontal regimes
}

G. Perret, A. Stegner, T. Dubos, Jean-Marc Chomaz, M. Farge

\section{To cite this version:}

G. Perret, A. Stegner, T. Dubos, Jean-Marc Chomaz, M. Farge. Stability of parallel wake flows in quasigeostrophic and frontal regimes. Physics of Fluids, 2006, 18 (12), pp.126602. 10.1063/1.2397563 . hal-01023371

\section{HAL Id: hal-01023371 \\ https://hal-polytechnique.archives-ouvertes.fr/hal-01023371}

Submitted on $20 \mathrm{Jul} 2014$

HAL is a multi-disciplinary open access archive for the deposit and dissemination of scientific research documents, whether they are published or not. The documents may come from teaching and research institutions in France or abroad, or from public or private research centers.
L'archive ouverte pluridisciplinaire HAL, est destinée au dépôt et à la diffusion de documents scientifiques de niveau recherche, publiés ou non, émanant des établissements d'enseignement et de recherche français ou étrangers, des laboratoires publics ou privés. 


\section{AIP $\left.\right|_{\text {Physics of }}$ Fluids}

\section{Stability of parallel wake flows in quasigeostrophic and frontal regimes}

G. Perret, A. Stegner, T. Dubos, J. M. Chomaz, and M. Farge

Citation: Physics of Fluids (1994-present) 18, 126602 (2006); doi: 10.1063/1.2397563

View online: http://dx.doi.org/10.1063/1.2397563

View Table of Contents: http://scitation.aip.org/content/aip/journal/pof2/18/12?ver=pdfcov

Published by the AIP Publishing

\section{Articles you may be interested in}

Transient growth of secondary instabilities in parallel wakes: Anti lift-up mechanism and hyperbolic instability Phys. Fluids 23, 114106 (2011); 10.1063/1.3659158

Investigation of the effect of external periodic flow pulsation on a cylinder wake using linear stability analysis Phys. Fluids 23, 094105 (2011); 10.1063/1.3625413

Large eddy simulation of the flow around single and two side-by-side cylinders at subcritical Reynolds numbers Phys. Fluids 23, 075101 (2011); 10.1063/1.3596267

Wake transition in flow past a circular cylinder

Phys. Fluids 22, 114104 (2010); 10.1063/1.3500692

Vortex shedding in high Reynolds number axisymmetric bluff-body wakes: Local linear instability and global bleed control

Phys. Fluids 16, 3460 (2004); 10.1063/1.1773071

\section{A|P| Journal of}




\title{
Stability of parallel wake flows in quasigeostrophic and frontal regimes
}

\author{
G. Perret ${ }^{a)}$ \\ Laboratoire de Mécanique Physique et Géoscience, Université du Havre, 25, rue Philippe Lebon, \\ 76600 Le Havre, France \\ A. Stegner ${ }^{\text {b) }}$ \\ Laboratoire de Météorologie Dynamique, CNRS-ENS, 24, rue Lhomond, 75231 Paris Cedex 05, France \\ T. Dubos \\ Laboratoire de Météorologie Dynamique, CNRS-Ecole Polytechnique, 91128 Palaiseau Cedex, France \\ J. M. Chomaz \\ Laboratoire d'Hydrodynamique-Ladhyx, CNRS-Ecole Polytechnique, 91128 Palaiseau Cedex, France \\ M. Farge \\ Laboratoire de Météorologie Dynamique, CNRS-ENS, 24, rue Lhomond, 75231 Paris Cedex 05, France
}

(Received 5 May 2006; accepted 20 October 2006; published online 12 December 2006)

\begin{abstract}
Recent laboratory experiments [G. Perret, A. Stegner, M. Farge, and T. Pichon, Phys. Fluids 18, 036603 (2006)] have shown that the vortex-street formed in the wake of a towed cylinder in a rotating shallow-water layer could present a strong cyclone-anticyclone asymmetry. In extreme cases, only large-scale anticyclones were observed in the far wake. This asymmetry occurs in the so-called frontal regime when the Rossby number is small and the surface deviation is large. This asymmetry may have various origins and in particular may be attributed to the asymmetry of the flow around the cylinder, to the linear stability property of the wake, or to its nonlinear evolution. To discriminate between these mechanisms, we study the stability of two idealized parallel flows in the quasigeostrophic and in the frontal regimes. These parallel flows correspond to two velocity profiles measured just behind the cylinder in a region where the perturbations are negligible. According to our linear stability analysis, the most unstable mode, in the frontal regime, is localized in the anticyclonic shear region whether the base flow profile is symmetric or not. On a linear basis, it is thus more the instability that imposes the asymmetry than the base flow. Direct numerical simulations of the synthetic parallel wake flows show that nonlinearity exacerbates the dominance of the anticyclonic mode linearly selected. By numerically studying the spatio-temporal evolution of a small perturbation localized in space, we show that, unlike incompressible two-dimensional wake flows and the symmetric wake in the quasigeostrophic regime, the parallel asymmetric wake is strongly convectively unstable in the frontal regime, and not absolutely unstable. When the surface deformation becomes large, the wake instability changes from the absolute instability in the quasi-geostrophic regime to the strongly convective instability of the frontal regime. This explains well the changes. (c) 2006 American Institute of Physics. [DOI: 10.1063/1.2397563]
\end{abstract}

\section{INTRODUCTION}

The dynamical origin of the classical two-dimensional von Karman street generated in the wake of an obstacle has been extensively studied. In contrast to this well-known pattern, wakes encountered in geophysical flows are affected by the Earth's rotation and the vertical stratification. In such cases, different types of cyclone-anticyclone asymmetries have been observed. ${ }^{1}$ The mechanisms underlying these asymmetries are an active research topic. On the one hand, for Rossby number larger than unity, $\operatorname{Ro}=V_{0} /(f R)$, where $V_{0}$ and $R$ are the characteristic velocity and length scale and $f$ is the Coriolis parameter, an inertial-centrifugal instability affects the anticyclonic vortices when the relative vorticity $\xi$ becomes smaller than $-f$. This three-dimensional instability is transient and the von Karman street becomes symmetric

\footnotetext{
${ }^{a)}$ Electronic mail: gaele.perret@univ-lehavre.fr

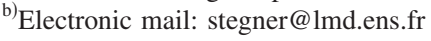

again further down the wake. On the other hand, in shallowwater flows, anticyclones tend to be predominant in the flow when the geopotential deformations are significant (large variations of the surface deviation). For finite Rossby numbers, large geopotential deviations are imposed by the cyclogeostrophic balance. ${ }^{2,3}$ For small Rossby numbers, the geopotential fluctuations can be significant for large-scale flows, i.e., when the local deformation radius $R_{d}$ is smaller than the size of the vortices, where $R_{d}=\sqrt{g H_{0}} / f, g$ is the gravity, and $H_{0}$ is the layer depth. In that case, anticyclonic vortices tend to be more robust ${ }^{4}$ and stable ${ }^{5-7}$ than cyclones. As far as large-scale wakes are concerned, a recent experimental study $^{8}$ shows that the vortex street greatly differs from the classical von Karman street. A strong cyclone-anticyclone asymmetry appears: anticyclones are circular whereas cyclones are stretched and deformed. For a specific range of parameters, only anticyclones are formed in the wake. However, no evidence of a selective destabilization of the cy- 
(a)

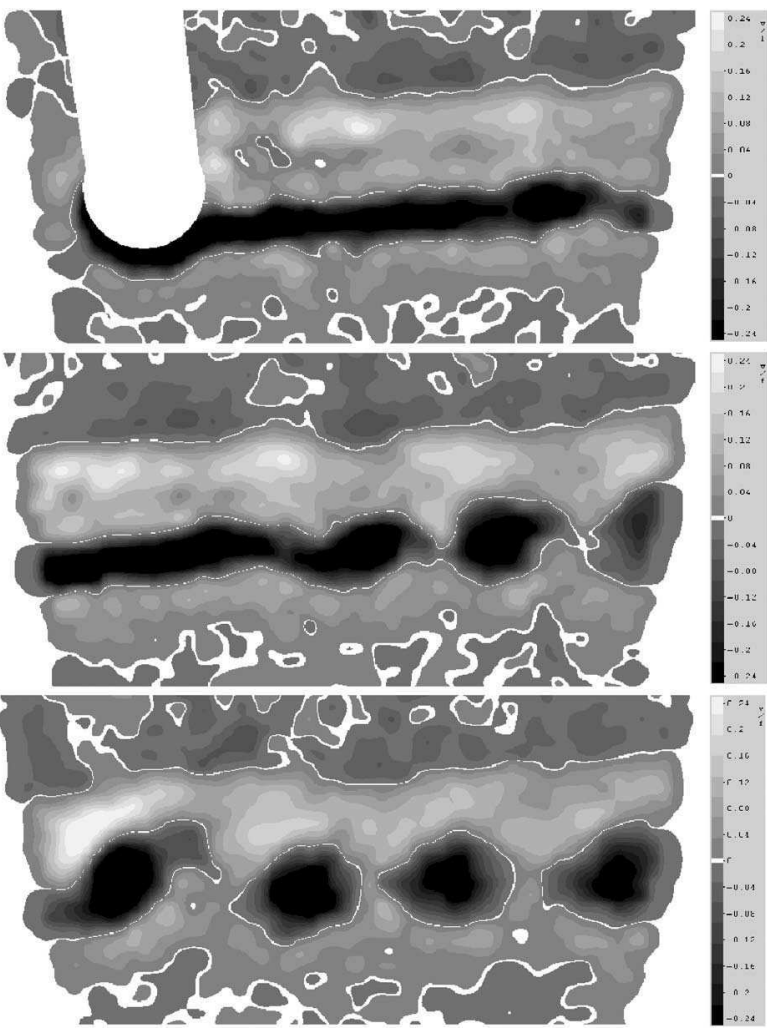

FIG. 1. Vorticity field of a large-scale cylinder wake observed in laboratory experiments at $T=1.3 T_{f}(\mathrm{a}), T=5 T_{f}(\mathrm{~b})$, and $T=10 T_{f}(\mathrm{c})$, where $T_{f}=2 \pi / f$ is the inertial period. The measurement area remains fixed while the cylinder is towed from right to left at a constant speed $V_{0}=0.57 \mathrm{~cm} \mathrm{~s}^{-1}$. The Rossby number is $\mathrm{Ro}=0.19$ and the Burger number is $\mathrm{Bu}=0.11$, where $\mathrm{Bu}$ $=\left(R_{d} / R\right)^{2}$ with $R$ the radius of the cylinder. The experimental setup and an equivalent color figure are given in Perret et al. (Ref. 8) The $x$ axis points to the left, the $y$ axis points down, and the anticyclones correspond to the dark region.

clonic vortices was observed once they are formed. Vortices are not shed just behind the obstacle as in the classical von Karman wake. Instead, a parallel steady wake flow extends up to several diameters behind the cylinder and only anticyclonic vortices roll up far downstream the wake (Fig. 1). The resulting flow pattern resembles more that of a mixing layer than a von Karman street. At the same time, the distance between equal sign vortices is reduced and the Strouhal number strongly increases. These quantitative observations suggest a change in the nature of the wake instability when the size of the cylinder becomes larger than the deformation radius. The main objective of the present study is to determine if the primary instability of the flow behind the cylinder that generates the vortex street could explain the cycloneanticyclone asymmetry observed experimentally in largescale wakes.

A key concept in the understanding of nonrotating wake instability is the concept of absolute versus convective stability of parallel flows. A parallel flow is said to be convectively unstable if a localized perturbation is amplified but advected downstream so that at any fixed station the perturbation finally relaxes back to zero. Conversely, a flow is absolutely unstable if a localized perturbation grows and contaminates the entire flow: at any fixed location, the per- turbation keeps increasing exponentially with time. In the absolute case, the nature of the instability changes in that the perturbation is able to propagate both upstream and downstream. For nonparallel flows, a global analysis is needed to determine the stability properties of the flow. However, when the flow varies slowly in the streamwise direction, i.e., in the WKB approximation, each streamwise location in the flow is considered locally parallel. The absolute or convective nature of the wake profile then depends on the streamwise location. In that context, Chomaz, Huerre, and Redekopp ${ }^{9}$ have shown that an absolutely unstable zone of finite extent is a necessary condition for a global mode to become self-excited. These results are confirmed by laboratory experiments. ${ }^{10-12}$ Experimental $^{13}$ and numerical studies ${ }^{14,15}$ showed that von Karman streets are a manifestation of a self-sustained oscillation (i.e., a global mode), associated to a local absolute instability region in the base flow. Hence a first step in order to understand the possible change in the nature of large-scale wake instability is to investigate the absolute versus the convective stability of local profiles.

Our approach consists in analyzing the linear and nonlinear stability of two experimental profiles measured from laboratory experiments described in Perret et al. ${ }^{8}$ for a quasigeostrophic and a large-scale configuration. The local stability analysis is particularly suitable since nonparallel effects seem to be weak for a wake in the frontal regime (Fig. 1). The dynamical regimes and the basic states are first presented in Sec. II.

Ripa's general criterion ${ }^{16}$ provides sufficient conditions for linear stability of rotating shallow-water parallel flows: if the potential vorticity is monotonic and the total change of particle velocity is smaller than the minimum phase speed of long gravity waves, then the flow is stable. However, for a wake flow the potential vorticity gradient always changes sign. Therefore, a full linear temporal stability analysis is needed. In Sec. III, we perform such an analysis to identify a possible cyclonic-anticyclonic selection in the linear stage of the instability. We especially analyze the spatial structure and the spanwise localization of the leading temporal modes. The nonlinear evolution of the perturbation is presented in Sec. IV. We investigate, in particular, whether the nonlinear saturation of the perturbed experimental profile can reproduce the asymmetric vortex street morphology observed in the experiment. Finally, we study in Sec. V. the linear impulse response to a localized disturbance to identify the convective or the absolute nature of the wake-flow instability. The conclusions are given in Sec. VI.

\section{PROBLEM FORMULATION}

We use the rotating shallow-water model on the $f$ plane, described in dimensionless form by the following system:

$$
\operatorname{Ro}\left(\frac{\partial \mathbf{V}}{\partial t}+(\mathbf{V} \cdot \nabla) \mathbf{V}\right)+\mathbf{n} \times \mathbf{V}=-\frac{\lambda \mathrm{Bu}}{\operatorname{Ro}} \nabla \eta+\frac{\mathrm{Ro}}{\operatorname{Re}} \nabla^{2} \mathbf{V}
$$




$$
\lambda\left(\frac{\partial \eta}{\partial t}+\mathbf{V} \cdot \nabla \eta\right)+(1+\lambda \eta) \nabla \cdot \mathbf{V}=0
$$

where $\mathbf{V}=(u, v)$ is the horizontal velocity scaled by the typical velocity $V_{0}, \eta$ is the dimensionless surface deviation, and $\mathbf{n}$ is the vertical unit vector of the $f$ plane. We define the Rossby number Ro, the relative surface deviation $\lambda$, the Burger number $\mathrm{Bu}$, and the Reynolds number Re as follows:

$$
\begin{aligned}
& \mathrm{Ro}=\frac{V_{0}}{f R}, \quad \lambda=\frac{\Delta h}{H_{0}}, \quad \mathrm{Bu}=\left(\frac{R_{d}}{R}\right)^{2} \quad \text { with } \\
& R_{d}=\frac{\sqrt{g H_{0}}}{f}, \quad \mathrm{Re}=\frac{2 V_{0} R}{\nu},
\end{aligned}
$$

where $f=2 \Omega$ is the Coriolis parameter, $R$ is the radius of the cylinder, $H_{0}$ is the mean layer depth, $\Delta h$ is the characteristic surface deviation considered here as a scaling parameter, which will be determined by dominant balance in a different regime, and $R_{d}$ is the deformation radius, with $g$ the gravity acceleration and $\nu$ the kinematic viscosity. The Rossby number is assumed small and fixed at Ro=0.1 in the whole study. Therefore, the geostrophic balance is satisfied at leading order, imposing the surface deviation to equilibrate the Coriolis term in Eq. (1). Thus, the dominant balance imposes $\lambda=\mathrm{Ro} / \mathrm{Bu}$, meaning that $\Delta h=R V_{0} f / g$.

Depending on the value of the Burger number, one distinguishes various dynamical regimes at small Rossby number. The classical quasigeostrophic model is defined when $\mathrm{Bu}=\mathcal{O}(1)$ or equivalently $\lambda=\mathcal{O}(\mathrm{Ro}) \ll 1$. In that limit, the symmetry $\vec{V} \rightarrow-\vec{V}$ and $\eta \rightarrow-\eta$ holds at the leading order, meaning that cyclones and anticyclones obey the same equation. Even if the Rossby number is not asymptotically small in this study $(\mathrm{Ro}=0.1)$, cyclones and anticyclones are expected to follow roughly the same evolution when $\mathrm{Bu}=\mathcal{O}(1)$. The second regime corresponds to the frontal regime and is defined by $\mathrm{Bu}=\mathcal{O}(\mathrm{Ro}) \ll 1$ or equivalently $\lambda=\mathcal{O}(1)$. In this regime, the flow is expected to follow at leading order the frontal geostrophic asymptotic model. ${ }^{17,18}$ In that model, the surface deviations are order unity, the symmetry $\vec{V} \rightarrow-\vec{V}$ and $\eta \rightarrow-\eta$ does not hold, and the evolution of cyclonic and anticyclonic structures should differ strongly.

According to laboratory experiments, a strong cycloneanticyclone asymmetry in the vortex street occurs for a cylinder wake in a frontal regime as shown in Fig. 1, where $\lambda=\mathrm{Ro} / \mathrm{Bu}=1.7$. In this regime, the experimental wake flow is observed to be quasiparallel over a distance of two diameters, as shown in Fig. 2, where the streamwise velocity profiles plotted for $x / R=0,1,2,3,4$ differ only slightly. In this quasiparallel shear zone, the streamwise velocity is asymmetric and exhibits an anticyclonic shear steeper than the cyclonic one. From this observation, one may wonder if the predominance of anticyclonic vortices is mainly due to the asymmetry of the velocity profile before it destabilizes or to the instability of the base flow in the frontal regime, i.e., a small deformation radius in comparison with the cylinder radius and thus the characteristic size of the wake.

By means of numerical studies, we can estimate the relative influence of the two effects: the asymmetry of the wake

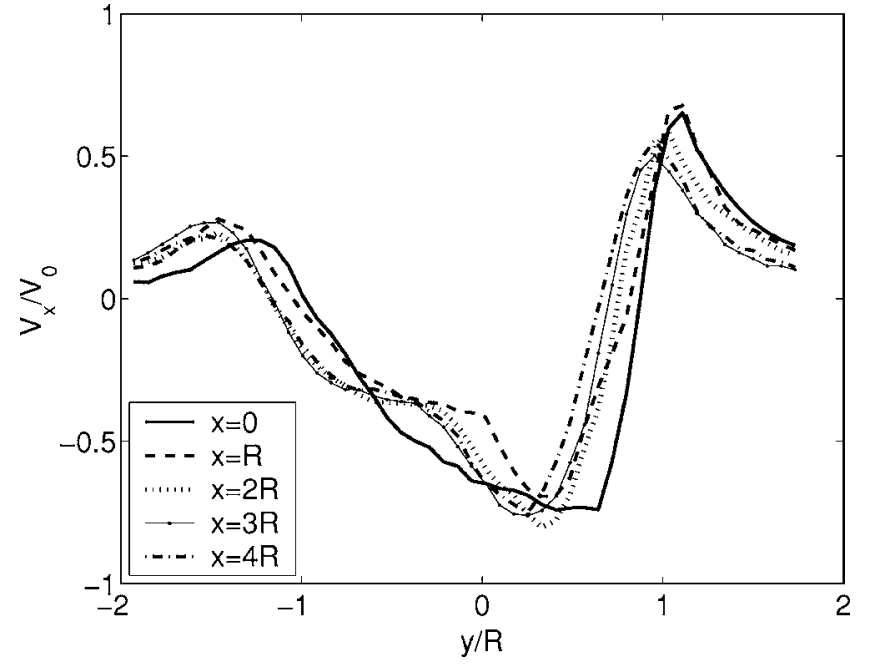

FIG. 2. Time-averaged velocity profiles, in the frontal regime, measured in the cylinder frame, at several locations in the extended double shear layer: just behind the cylinder and at one, two, three, and four radii behind.

profile versus the dynamical regime on the stability property of the flow. To do this, we dissociate the velocity profile from the parameter set of the experiment in which the profile was measured. Then, for each velocity profile (symmetric or asymmetric) we can study its stability in different regimes (quasigeostrophic or frontal geostrophic) by changing the value of the Burger number. Hence, in what follows, we study the stability of the symmetric wake flow both in a frontal $(\lambda=1.0)$ and a quasigeostrophic regime $(\lambda=0.1)$, and the stability of the asymmetrical parallel wake flow for the same two parameter sets. We thus consider four cases. In the case of the destabilization of a parallel wake flow in a quasigeostrophic regime $(\mathrm{Ro}=\lambda=0.1, \mathrm{Bu}=1)$, we anticipate that the cyclone-anticyclone asymmetry will be present for the asymmetric velocity profile. In the case of the destabilization of a symmetric parallel wake flow in a frontal regime (Ro $=0.1, \lambda=1.0, \mathrm{Bu}=0.1)$, the cyclone-anticyclone asymmetry will be induced only by the instability dynamics in that regime. As already stated, the symmetric and the asymmetric velocity profiles have been measured in two different experiments at a single location, close behind the cylinder, where no vortex has formed yet. In order to filter out the waves, they are averaged in time over the wave period, which is a tenth of the vortex shedding period. The symmetric wake was measured from a laboratory experiment performed in a quasigeostrophic regime at $\mathrm{Bu}=0.67, \mathrm{Ro}=0.06$, and $\mathrm{Re}$ $=200[$ Fig. $3(\mathrm{a})]$ and the asymmetric wake from an experiment performed in a frontal regime at $\mathrm{Bu}=0.11, \mathrm{Ro}=0.19$, and $\mathrm{Re}=800$ [Fig. 3(b)]. We emphasize that although these profiles are generated towing a cylinder at specific values of the control parameters, the measured local velocity profiles define a parallel flow solution of the shallow-water equations valid for all values of the control parameters. Thus, their stability may be considered for any value of the control parameters, independently of the specific one at which they have been experimentally measured. This remark is essential in order to realize that the asymmetry in the basic state and the asymmetry in the stability properties may be addressed 

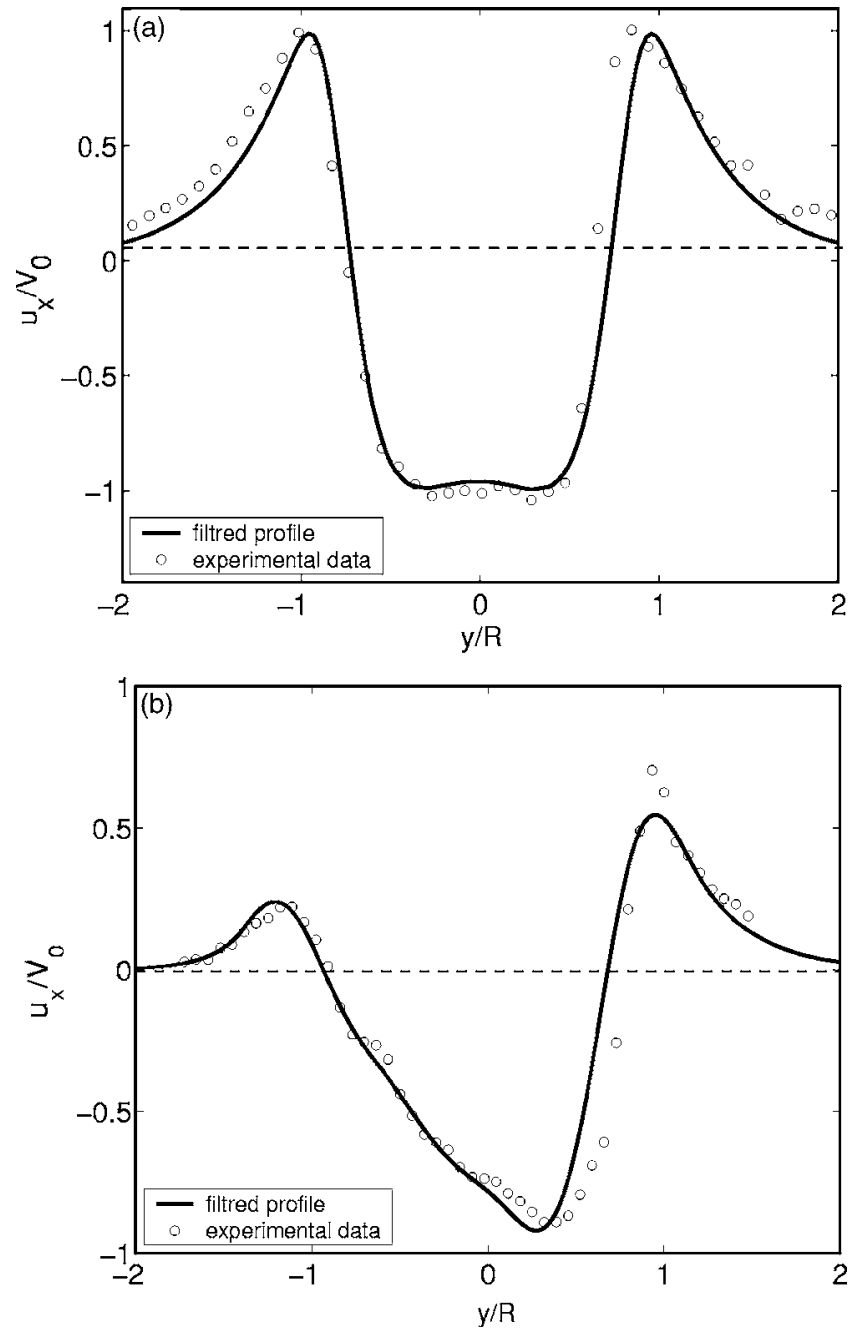

FIG. 3. Symmetrical (a) and asymmetrical (b) velocity profiles (solid line) filtered and interpolated from experimental profiles (dots).

separately. To analyze their stability, the experimental profiles are smoothed with a lowpass Fourier filter and interpolated to $N_{y}=256$ or $N_{y}=512$ points to match the resolution of the linear stability solver. Modifications caused by this procedure are minor, as is visible in Fig. 3. The maximum of the velocity profile is not exactly localized at $y / R=1$ and -1 since the original scaling based on the cylinder radius has been kept. The basic state surface deviation $\bar{\eta}$ is then calculated according to the geostrophic balance relation, $\bar{U}=-d \bar{\eta} / d y$.

\section{LINEAR STABILITY ANALYSIS}

In this section, we perform a temporal stability analysis of the parallel flows corresponding to the profiles plotted in Fig. 3. We assume that the underlying instability is inviscid and therefore we consider the rotating shallow-water system [Eqs. (1) and (2)] without viscous terms. The governing equations are linearized around a parallel basic state $(\bar{U}(y), \bar{\eta}(y))$. The perturbation field is expanded in normal form, $\quad(\widetilde{u}(x, y, t), \widetilde{v}(x, y, t), \widetilde{\eta}(x, y, t))=(u(\hat{y}), v(\hat{y})$, $\eta(\hat{y})) e^{i(k x-\omega t)}$, with $\omega=\omega_{r}+i \sigma$ the complex eigenfrequency and $k$ the real wave number. We then obtain the following eigenvalue problem:

$$
\underbrace{\mathcal{L}}_{\left.\begin{array}{ccc}
-k \frac{\partial \bar{\eta}}{\partial y} & i\left(\frac{\partial^{2} \bar{\eta}}{\partial y^{2}}+\mathrm{Ro}^{-1}\right) & \mathrm{Ro}^{-1} k \\
-i \mathrm{Ro}^{-1} & -k \frac{\partial \bar{\eta}}{\partial y} & i \mathrm{Ro}^{-1} \frac{\partial}{\partial y} \\
\left(\lambda^{-1}+\bar{\eta}\right) k & -i\left(\frac{\partial \bar{\eta}}{\partial y}+\left(\lambda^{-1}+\bar{\eta}\right) \frac{\partial}{\partial y}\right) & -k \frac{\partial \bar{\eta}}{\partial y}
\end{array}\right)}
$$

The matrix of the linear operator $\mathcal{L}$ is computed in a spectral basis with periodic boundary conditions and diagonalized using the LAPACK linear algebra package. ${ }^{19}$ The error in the growth rate $\sigma$ for a resolution of 512 and 256 is err $=\Sigma_{k}\left(\sigma_{512}-\sigma_{256}\right)^{2} / \Sigma_{k} \sigma_{512}^{2}=9.2 \times 10^{-5}$. This implies that a resolution of 256 collocation points in the spanwise direction is sufficient to perform the linear simulations.

Figure 4 presents the leading unstable modes of the symmetric velocity profile [Fig. 3(a)] for $\lambda=0.1$ and $\lambda=1$, the Rossby number being the same $\mathrm{Ro}=0.1$. The perturbation energy $\delta E$, with $\delta E=\left(H_{0}+\bar{\eta}\right)\left(\hat{u}^{2}+\hat{v}^{2}\right) / 2+\hat{\eta}(\hat{\eta} / 2+\bar{U} \hat{u})$, of the two most unstable eigenmodes is plotted in Figs. 4(e) and 4(f) for a quasigeostrophic regime and a frontal regime for all the cases. The most unstable eigenmode corresponds to a perturbation energy preferentially localized in the anticyclonic shear region of the base flow. This mode is called the anticyclonic, denoted mode $\mathrm{A}$ and plotted as the solid line in Figs. 4(e) and 4(f). The second mode corresponds to perturbation energy essentially localized in the cyclonic region. This mode is called the cyclonic mode by extension, denoted mode C, and plotted as the dashed line in Figs. 4(e) and 4(f). The relative potential vorticity $(\mathrm{PV})$ of the basic state is plotted as a reference in Figs. 4(c) and 4(d) for the two regimes. It shows that, although the vorticity profile is symmetric, this is not the case for the PV profile when $\lambda=\mathrm{Ro} / \mathrm{Bu}$ is not small. The maximum of perturbation energy for the anticyclonic mode (cyclonic mode) is localized at the minimum (maximum) of the potential vorticity basic state.

In the quasigeostrophic regime, i.e., at $\lambda=0.1$, the growth rates of modes $\mathrm{A}$ and $\mathrm{C}$ for the symmetric wake are very close [Fig. 4(a)]. The slight difference is due to small ageostrophic effects since the Rossby number in our simulations is small but finite, Ro=0.1. Calculations (not shown) performed with a quasigeostrophic model $(\mathrm{Ro} \rightarrow 0)$ using the same stability solver showed that both growth rates then do coincide.

In the frontal regime, at $\lambda=1.0$, the growth rates of the two most unstable modes differ greatly. While the anticyclonic growth rate remains close to its quasigeostrophic 

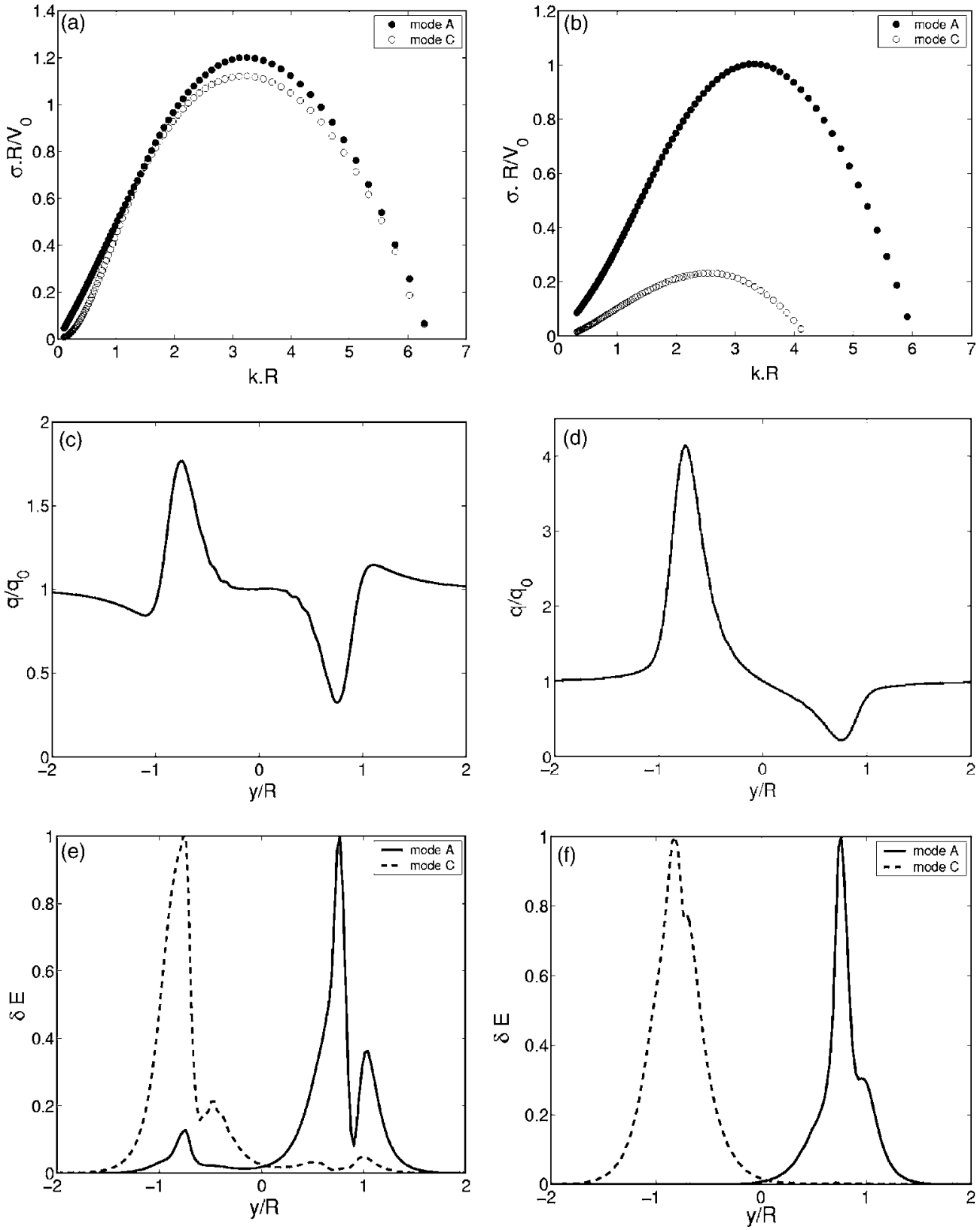

FIG. 4. Growth rate of the two most unstable branches for the symmetrical wake profile [Fig. 3(a)] in a quasigeostrophic regime ( $\lambda=0.1)$ (a) and frontal regime $(\lambda=1.0)$ (b). Potential vorticity of the unperturbed wake flow in a quasigeostrophic regime (c) and a frontal regime (d). Spatial localization, in terms of energy, of the anticyclonic mode (solid line) and the cyclonic mode (dashed line) in a quasigeostrophic regime for $k R=3.24$ (e) and a frontal regime for $k R=3.52$ (f).

value, the growth rate of the cyclonic mode drops. The basic PV state is strongly asymmetric with an absolute value which is much larger in the cyclonic than in the anticyclonic shear. Anticyclonic and cyclonic modes are fully separated in space and localized in the anticyclonic and in the cyclonic shear, respectively [Fig. 4(f)]. In contrast, in the quasigeostrophic regime $(\lambda=0.1)$ the anticyclonic mode (cyclonic mode) is localized in both shears with a larger value in the anticyclonic (cyclonic) region. Thus even though the velocity profile is symmetric in a frontal regime $(\lambda=1.0)$, the most unstable mode affects only the anticyclonic shear.

Figure 5 is the same as Fig. 4 but for the asymmetric velocity profile. Figures 5(a) and 5(b) show the growth rates of the two most unstable modes for $\lambda=0.1$ and 1.0. In a quasigeostrophic regime, the growth rate for the anticyclonic branch is already much larger than for the cyclonic one. This is consistent with a Kelvin Helmoltz-type instability if we consider the two shear layers as dissociated. Indeed, if we neglect the coupling between the two shear layers, each branch corresponds to the Kelvin Helmoltz instability of a single shear layer known to induce instability, with the growth rate scaling with the shear $\Delta U / d$, where $\Delta U=U_{\max }$ $-U_{\min }$ and $d$ is the distance between maximum and minimum velocity. The shear is three times larger in the anticyclonic part of the flow $(\Delta U / d=2.22)$ than in the cyclonic part $(\Delta U / d=0.77)$ and the maximum growth rate ratio is indeed about 3. In a frontal regime $(\lambda=1.0)$, the growth rate difference increases: the maximum growth rate decreases by 

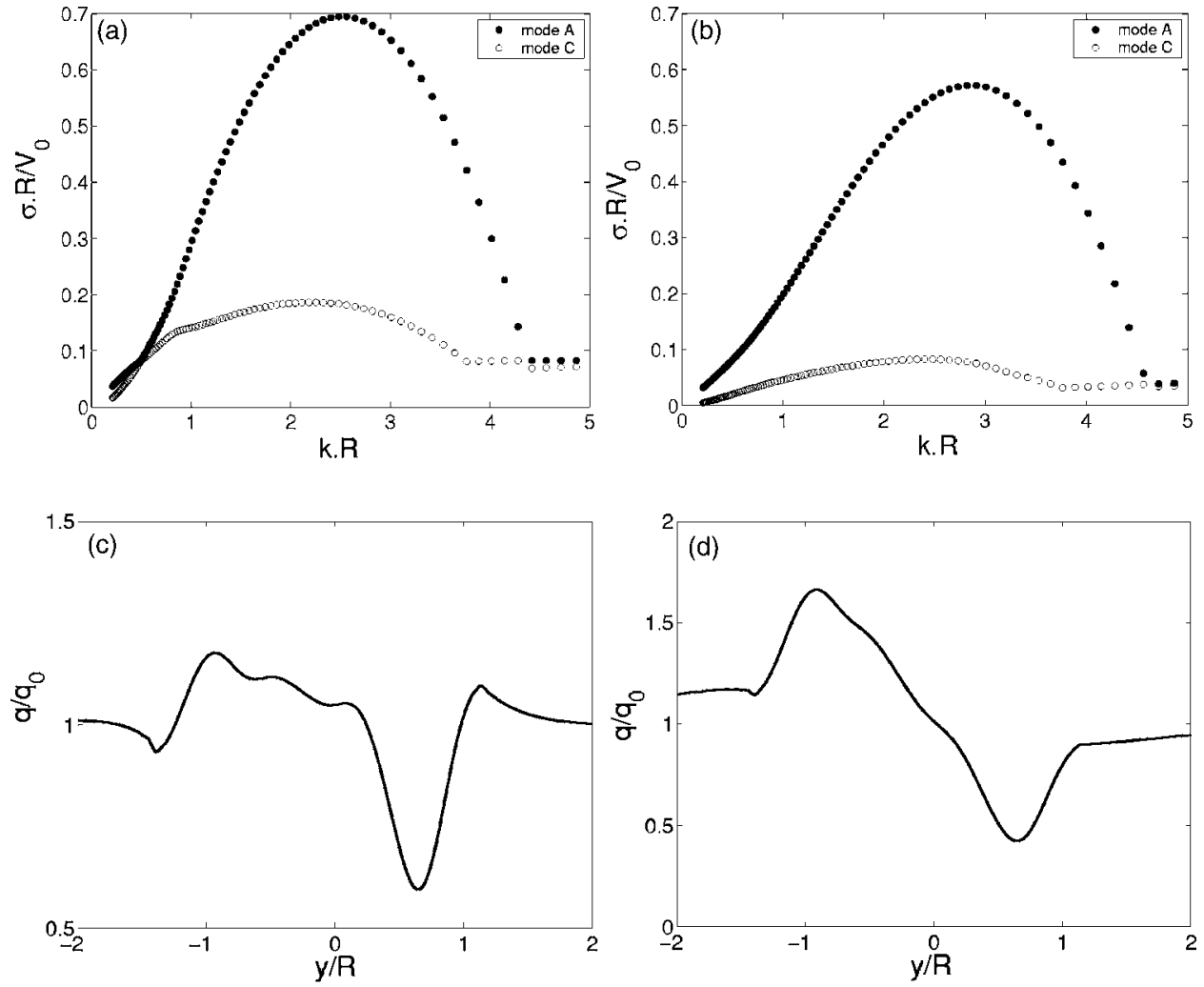

FIG. 5. Same as Fig. 4 but for the asymmetric profile [Fig. 3(b)].
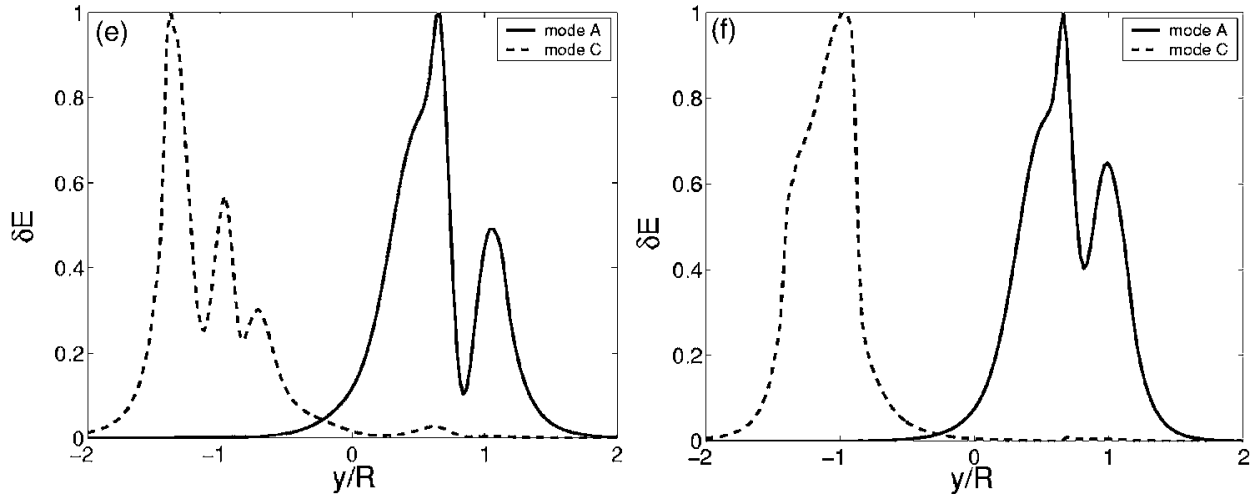

$20 \%$ for the anticyclonic mode and by $80 \%$ for the cyclonic mode. Figures 5(e) and 5(f) show the perturbation energy $\delta E$ of the two most unstable eigenmodes in the quasigeostrophic and frontal regimes. Each mode develops quite exclusively in one part of the flow, so that the development of the leading instability, mode A, is essentially localized in the anticyclonic shear, even in a quasigeostrophic regime $(\lambda=0.1)$. As in the symmetric basic state, the maximum of perturbation energy is localized at the extremum of the basic state PV [Figs. 5(c) and 5(d)]. Note that in a frontal regime, the PV profile actually relaxes to 1 at the end of the domain $(y / R$ $= \pm 3$ ), not shown in the figure. To sum up, the frontal regime tends to favor the development of instability in the anticyclonic shear independently of the velocity profile of the base flow. In the linear stage of the instability at least, cyclonic and anticyclonic shears have different stability properties. The maximum growth rates and the corresponding wave numbers for the four cases are summarized in Table I.

\section{NONLINEAR EVOLUTION}

The nonlinear evolution of the instability, the cycloneanticyclone interaction, and the resulting vortex-street pattern are studied by computing the nonlinear evolution of the perturbed parallel flows corresponding to the velocity profiles discussed previously. For each parallel wake profile, the nonlinear stability evolution is computed in the quasigeostrophic $(\lambda=0.1)$ and the frontal regime $(\lambda=1.0)$. The rotating shallow-water equations are discretized in space with a pseudospectral method and in time with a second-order leapfrog scheme. In order to follow the propagation of wavepacket fronts (discussed in Sec. V), we use a long rectangular box with a resolution $256 \times 1024 \approx 6 \times 24$. The basic state is parallel with the same velocity profile extended to the entire domain and the boundary conditions are periodic. To reduce viscous effects, the Reynolds number is fixed at 5000. When a numerical simulation is initialized with a parallel basic 
TABLE I. Maximum growth rate $\left(\sigma_{\max }\right)$ and corresponding wave number $\left(k_{\max }\right)$ for the anticyclonic branch (mode A) and the cyclonic branch (mode C) in different regimes: quasigeostrophic $(\lambda=0.1)$ and frontal $(\lambda=1.0)$.

\begin{tabular}{cccccc}
\hline \hline & & \multicolumn{2}{c}{ Symmetric wake } & \multicolumn{2}{c}{ Asymmetric wake } \\
\cline { 3 - 6 } & & $\lambda=0.1$ & $\lambda=1.0$ & $\lambda=0.1$ & $\lambda=1.0$ \\
\hline \multirow{2}{*}{$\frac{\sigma_{\max } R}{V_{0}}$} & mode A & 1.20 & 1.11 & 0.69 & 0.57 \\
\multirow{2}{*}{$k_{\max } R$} & mode C & 1.12 & 0.28 & 0.19 & 0.08 \\
& mode A & 3.24 & 3.52 & 2.48 & 2.82 \\
& mode C & 3.24 & 2.86 & 2.18 & 2.40 \\
\hline \hline
\end{tabular}

velocity profile, without external perturbation, the flow remains parallel and the velocity diffuses slowly. The basic state is then initially perturbed with a small, localized perturbation consisting of two symmetric dipoles parallel to the $y$ axis with a Gaussian envelope, located at the maximum absolute value of vorticity in both cyclonic and anticyclonic shear. This perturbation is similar to that used by Chomaz, ${ }^{20}$

$$
\begin{aligned}
\phi= & g \widetilde{\eta} \propto \sin \left(\frac{x-x_{0}}{\delta}\right) \\
& \times\left(e^{-\left(x-x_{0}\right)^{2}+\left(y-y_{0}\right)^{2} / \delta^{2}}+e^{-\left(x-x_{0}\right)^{2}+\left(y-y_{1}\right)^{2} / \delta^{2}}\right)
\end{aligned}
$$

with $x_{0}=12$ the center of the dipoles in the streamwise direction, $y_{0}=1$ and $y_{1}=-1$ the center of each dipole in the spanwise direction, and $\delta=3 \Delta x=0.07$ the initial size of the perturbation. The velocity is then adjusted to the geostrophic balance in order to avoid gravity wave emission.

As expected for $\lambda=0.1$, i.e., in a quasigeostrophic regime, the nonlinear saturation of the instability for the symmetric wake flow does not induce an asymmetry [Fig. 6(a)]. The vortex street is symmetric: cyclones and anticyclones have similar circular shape and strength with opposite extrema of relative vorticity $\xi_{\max }=-\xi_{\min }=0.45$, where $\xi$ is the relative vorticity (we recall that the time has been nondimensionalized such that the Coriolis parameter is $f=1$ ). However, the vortex street formed by the nonlinear evolution of the instability [Fig. 6(a)] is very different from that observed in laboratory experiment [Fig. 6(e)] in the same dynamical regime. The size of vortices and the distance between same sign vortices $d_{0}$ are much smaller than in the experiment. For example, $d_{0}$ in the experiment is about $6.7 R$ whereas it is only $2 R$ for the nonlinear development of instability. The Strouhal number is also very different in nonlinear stability analysis and laboratory experiments. For the nonlinear numerical simulations, the Strouhal number is estimated as follows: $\mathrm{St}=\left(1-V_{d} / V_{0}\right) 2 R / d_{0}$, where $2 R$ is the cylinder diameter. The drift velocity $V_{d}$ of vortices and the distance between two same sign vortices $d_{0}$ are computed from the simulations. One may note that the distance between two same sign vortices is equal to the wavelength of the most unstable linear mode, mode $\mathrm{A}, \lambda_{\max }=2 \pi / k_{\max }$. As for the drift velocity of vortices $V_{d}$, it differs slightly (by $4 \%$ ) from the phase velocity of the most unstable mode $\omega_{r} / k_{\max }$ with $\omega_{r}$ the real frequency, so that a Strouhal number calculated from linear analysis $\mathrm{St}=\left[1-\omega_{r} /\left(k_{\max } V_{0}\right)\right] 2 R / \lambda_{\max }$ also differs by only $4 \%$ from the Strouhal number estimated from nonlinear simulations. Using the above definition, the Strouhal number in nonlinear simulations is $\mathrm{St}=0.96$, whereas it is only $\mathrm{St}=0.3$ in the laboratory experiment. A possible reason for this strong discrepancy is that a purely local temporal stability analysis is sufficient only for flows that are strictly parallel, which is not the case of the present experimental flow. Indeed, for the classical von Karman street one has to consider the global instability to predict the Strouhal number. ${ }^{9,21}$ In particular, the frequency selection is imposed by the wave of zero group velocity and not by the most unstable wave. Therefore, the pattern selection of temporally evolving parallel wake simulations in a quasigeostrophic regime differs strongly from the spatially evolving real wake.

Next we compute the nonlinear evolution of the symmetric parallel wake in the frontal regime, corresponding to $\lambda=1.0$ [Fig. 6(b)]. In this case, even though the basic velocity profile is symmetric the perturbations evolve into a strongly asymmetric vortex street. Anticyclones remain almost circular, whereas cyclones are greatly deformed and stretched. This result is consistent with the linear stability analysis, which shows that the most unstable mode is fully localized in the anticyclonic part of the flow. The nonlinear interactions seem to reinforce this initial asymmetry. A recent study by Graves, McWilliams, and Montgomery ${ }^{3}$ exhibits a new mechanism of vortex weakening under straining deformation in the framework of rotating shallow-water flow. When the vortex radius is larger than the deformation radius and the Rossby number is finite, the strain-induced vortex weakening is greater for cyclones than anticyclones. Such nonlinear vortex-vortex interaction could reinforce the initial asymmetry given by the linear stability analysis. Even if this symmetric velocity profile with large geopotential deviation cannot be generated by towing a cylinder, this numerical simulation emphasizes that anticyclonic predominance is intrinsic to the linear and nonlinear stability properties of the frontal regime only.

Likewise, the asymmetric wake profile in a quasigeostrophic regime would not be encountered in the wake of a real obstacle. Nevertheless, we study its nonlinear evolution in order to qualitatively estimate the asymmetry induced by the velocity profile alone. This case corresponds to Fig. 6(c). The vortices of both signs that emerge in the wake have comparable sizes but different intensities. Consistent with the asymmetry of the basic velocity profile, anticyclones have 


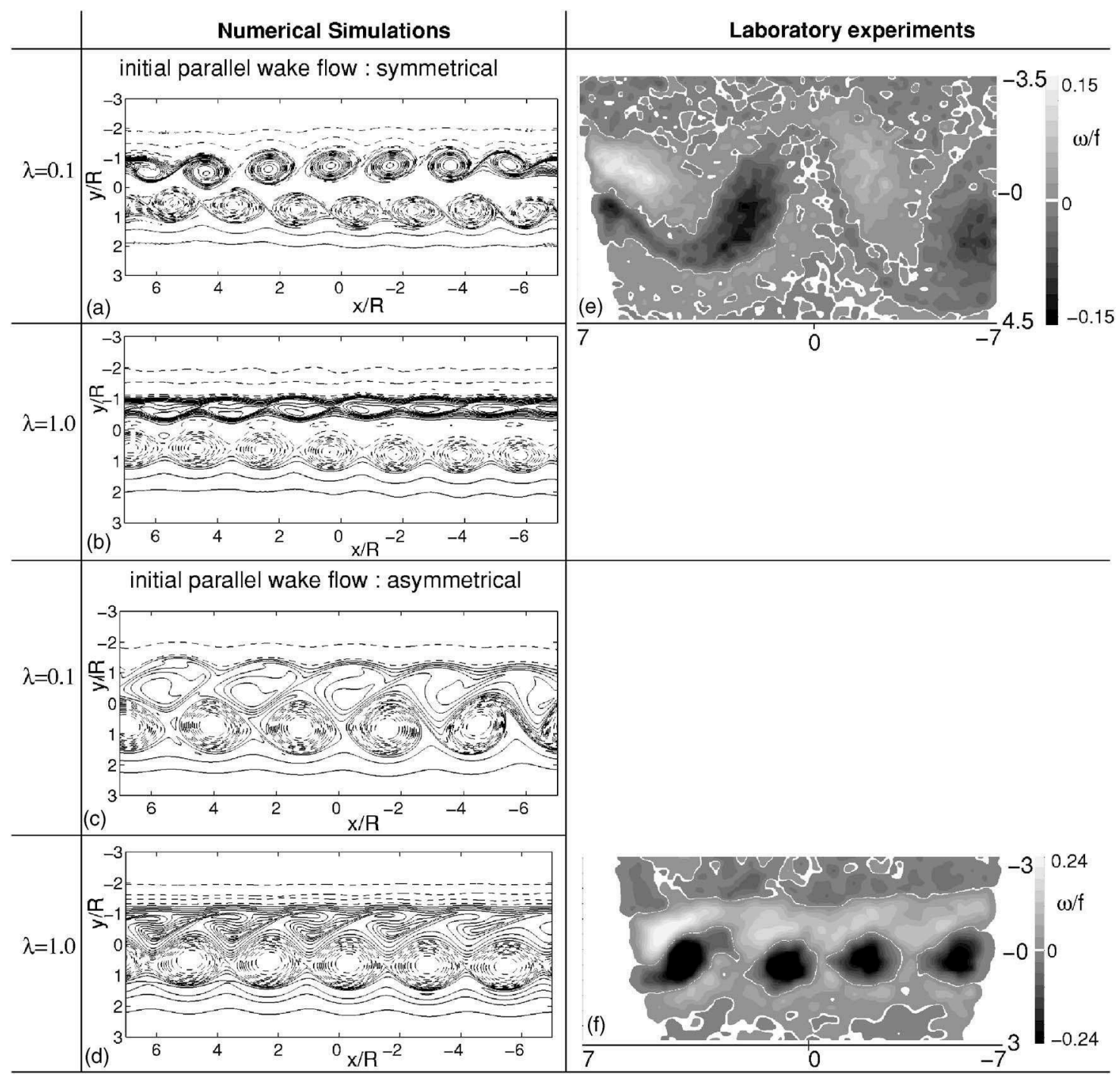

FIG. 6. Nonlinear evolution of the computed vorticity field for the symmetrical wake profile [Fig. 3(a)] at $T=12 \tau$, where $\tau=D / V_{0}$ for $\lambda=0.1$ (a) and $T=30 \tau$ for $\lambda=1.0$ (b), and for the asymmetrical wake profile [Fig. 3(b)] at $T=20 \tau$ for $\lambda=0.1$ (c) and $T=64 \tau$ for $\lambda=1.0$ (d). Solid line contours correspond to positive vorticity (cyclones) and dashed line contours to negative vorticity (anticyclones). Vorticity field obtained by PIV in laboratory experiments for Ro $=0.06$ and $\lambda=0.09$ (e) and $\mathrm{Ro}=0.19$ and $\lambda=1.7$ (f) are plotted for comparison.

higher vorticity than their cyclonic counterparts. Despite their weak intensity in comparison with anticyclones, cyclones are less stretched than in the previous symmetric case in a frontal regime.

Finally we present the nonlinear evolution of the asymmetric wake in a frontal regime $(\lambda=1)$ [Fig. 6(d)]. This case does correspond to a real experimental configuration [Fig. 6(f)]. The difference with the previous case [Fig. 6(c)] is only due to the change in the parameter $\lambda$, i.e., to the dynamical regime. We observed that cyclones are strongly stretched and present a boomerang shape. Hence, for an asymmetric wake flow, the cyclone-anticyclone asymmetry is greatly enhanced by the frontal regime. Note that similar boomerang-shaped structures were also observed by Poulin and Flierl $^{22}$ for Rossby number of order 1 . Although their study corresponds to a different range of parameters, they have also reported large geopotential deviations in the flow indicating that $\lambda$ might be the key parameter that explains the similarity of the flow patterns. In the frontal regime, we com- pare the nonlinear evolution of the perturbation, where $\lambda=1.0$, with the vorticity field observed in laboratory experiment for $\lambda=1.7$ [Fig. 6(f)]. It is quite remarkable that the pattern arising from the nonlinear instability of the parallel flow reproduces the general pattern of the vortex street observed far behind the cylinder in the experiment. Indeed, both the shape and the intensities of the coherent structures that emerge in the nonlinear simulation are in good agreement with the experimental measurements. Moreover, the Strouhal number estimated from the parallel flow analysis $(\mathrm{St}=0.71$ calculated from the nonlinear simulations and $\mathrm{St}=0.76$ calculated from the linear analysis) has the same order as measured in the experiment $(\mathrm{St}=0.56)$. In addition, if we use for our simulation exactly the same parameters as in the experiment $(\operatorname{Re}=800, \operatorname{Ro}=0.19$, and $\lambda=1.7)$, we obtain an even better agreement on the Strouhal number: $\mathrm{St}$ $=0.66$.

Despite its local character, the nonlinear stability analysis, in the frontal regime, recovers the vortex street pattern 
and the Strouhal number of the laboratory experiment. The similarity between the temporal evolution of the parallel wake and the spatial evolution of real wake suggests that the vortex street formed in the laboratory experiment is due to the noise driven destabilization of the quasiparallel wake extending behind the cylinder rather than the emergence of a global mode as in the classic von Karman street. If so, the instability should be a shear layer-type instability, convective, rather than a wake-type instability, which is absolute in a region of finite extent. To determine a change in nature of the instability, we now investigate the absolute or convective character of the present wake instability since this property is believed to determine the global stability of quasiparallel real open flows.

\section{ABSOLUTE/CONVECTIVE INSTABILITY}

In order to determine the absolute/convective character of the instability in real experiments, the study is, from now on, restricted to the analysis of the linear spatio-temporal development of the wave packet for the two physical cases: the symmetric wake for $\lambda=0.1$ in a quasigeostrophic regime and the asymmetrical wake for $\lambda=1.0$ in a frontal regime. The analysis performed in a quasigeostrophic (frontal) regime then corresponds to the symmetric (asymmetric) wake flow analysis. In the experiments, the cylinder was towed from right to left with a constant velocity $\mathbf{V}_{\mathbf{0}}$. The analysis is performed in the frame of the cylinder as in the nonlinear numerical simulations (Sec. IV), in the absence of external perturbations, the base flow remains parallel and diffuses slowly. The diffusion time is assumed long enough for the quasisteady approximation to apply. A localized perturbation [Eq. (3)] is initially introduced with a very small amplitude in order to remain in the linear regime until the end of the simulation.

We plot in Fig. 7 the spatio-temporal evolution of the spanwise-integrated enstrophy $\zeta(x, t)$ of the wave packet initially localized at $x_{0}=12 R$, with

$$
\zeta(x, t)=\int_{-L_{y} / 2}^{L_{y} / 2} \xi(x, y, t)^{2} d y,
$$

where $\xi$ is the vorticity of the perturbation and $L_{y}$ is the size of the domain in the spanwise direction. The absolute or convective character of the instability is given by the evolution of the perturbation at its initial streamwise position $x=x_{0}$. If the perturbation grows indefinitely at $x=x_{0}$, the instability is absolute, otherwise it is convective. More generally, theory predicts the long-time behavior along the fictitious trajectory $x(t)=x_{0}+v_{g} t, 23$

$$
\xi\left(x_{0}+v_{g} t, y, t\right) \propto t^{-1 / 2} e^{i\left[k\left(v_{g}\right)\left(x-x_{0}\right)-\omega\left(k\left(v_{g}\right)\right) t\right]}, \quad t \rightarrow \infty,
$$

where $\omega(k)$ is the dispersion relation between the complex streamwise wave number $k$ and the complex frequency $\omega=\omega_{r}+i \sigma$, and $k\left(v_{g}\right)$ is such that the group velocity $\mathrm{d} \omega / \mathrm{d} k$ at $k\left(v_{g}\right)$ equals $v_{g}$ (see Delbende $e t a l .{ }^{24}$ for the detailed theory).

Therefore, in order to determine this asymptotic behavior, we redrew the temporal evolution of $\zeta(x, t)$ as a function of the group velocity $v_{g}=\left(x-x_{0}\right) / t$, with $x-x_{0}$ the relative coordinate in the streamwise direction, $x_{0}$ being the initial

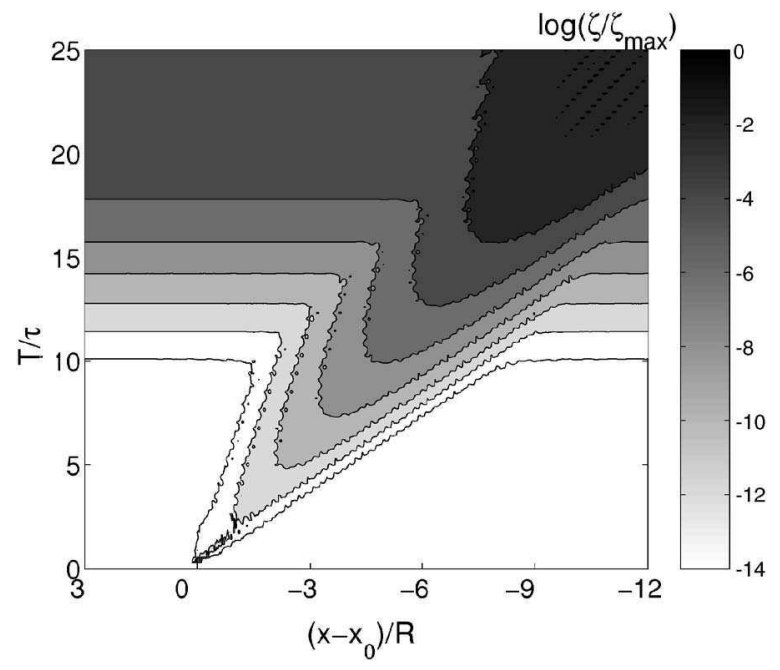

FIG. 7. Spatio-temporal evolution of a wave-packet perturbation initially localized at $x=x_{0}$ within the parallel asymmetric wake flow in the frontal regime. This graph is set in the cylinder frame moving from right to left and the gray scale corresponds to the logarithm of the amplitude of the spanwise integrated enstrophy.

position of the perturbation [Fig. 8 (circles)]. However, at a given time each curve presents important oscillations due to the wavy shape of the real perturbation. In order to get rid of this oscillation, we perform a spatial demodulation of the signal through a Hilbert transform: in practice, we Fourier transform the perturbation vorticity, cancel the Fourier coefficients corresponding to negative wave numbers, perform an inverse Fourier transform, and obtain $\hat{\xi}(x, y, t)$, whose modulus is the smooth envelope of the wave packet. The corresponding spanwise-integrated enstrophy is then monotonic at long times,

$$
\tilde{\zeta}\left(v_{g}, t\right)=\int_{-L_{y} / 2}^{L_{y} / 2}\left|\xi\left(x_{0}+v_{g} t, y, t\right)\right|^{2} d y \propto t^{-1} e^{2 \sigma\left(v_{g}\right) t} .
$$

We plot $\log \left[t \widetilde{\zeta}\left(v_{g}, t\right)\right]$ in Fig. 8 (solid lines) as a function of the group velocity $v_{g}=\left(x-x_{0}\right) / t$ at different instants for the symmetric wake in a quasigeostrophic [Fig. 8(a)] and the asymmetric wake in a frontal regime [Fig. 8(b)]. The two vertical lines indicate the group velocity of the trailing (left) and leading (right) edges of the wave packet where $\sigma\left(v_{g}\right)$ vanishes.

A numerical estimate of the spatio-temporal growth rate $\sigma$ is

$$
\sigma\left(v_{g}\right) \approx \frac{1}{2\left(t_{2}-t_{1}\right)} \ln \frac{t_{2} \widetilde{\zeta}\left(x_{0}+v_{g} t_{2}, t_{2}\right)}{t_{1} \widetilde{\zeta}\left(x_{0}+v_{g} t_{1}, t_{1}\right)} .
$$

Hence this method, detailed in Delbende et al., ${ }^{24}$ directly relates the group velocity $v_{g}$ and the growth rate $\sigma$. It presents an efficient alternative to the traditional pinch point search that would have been possible by extending the temporal stability solver used in Sec. III, which deals with real wave numbers and complex group velocities, to spatiotemporal stability analysis, which deals with real group velocities and complex wave numbers. 

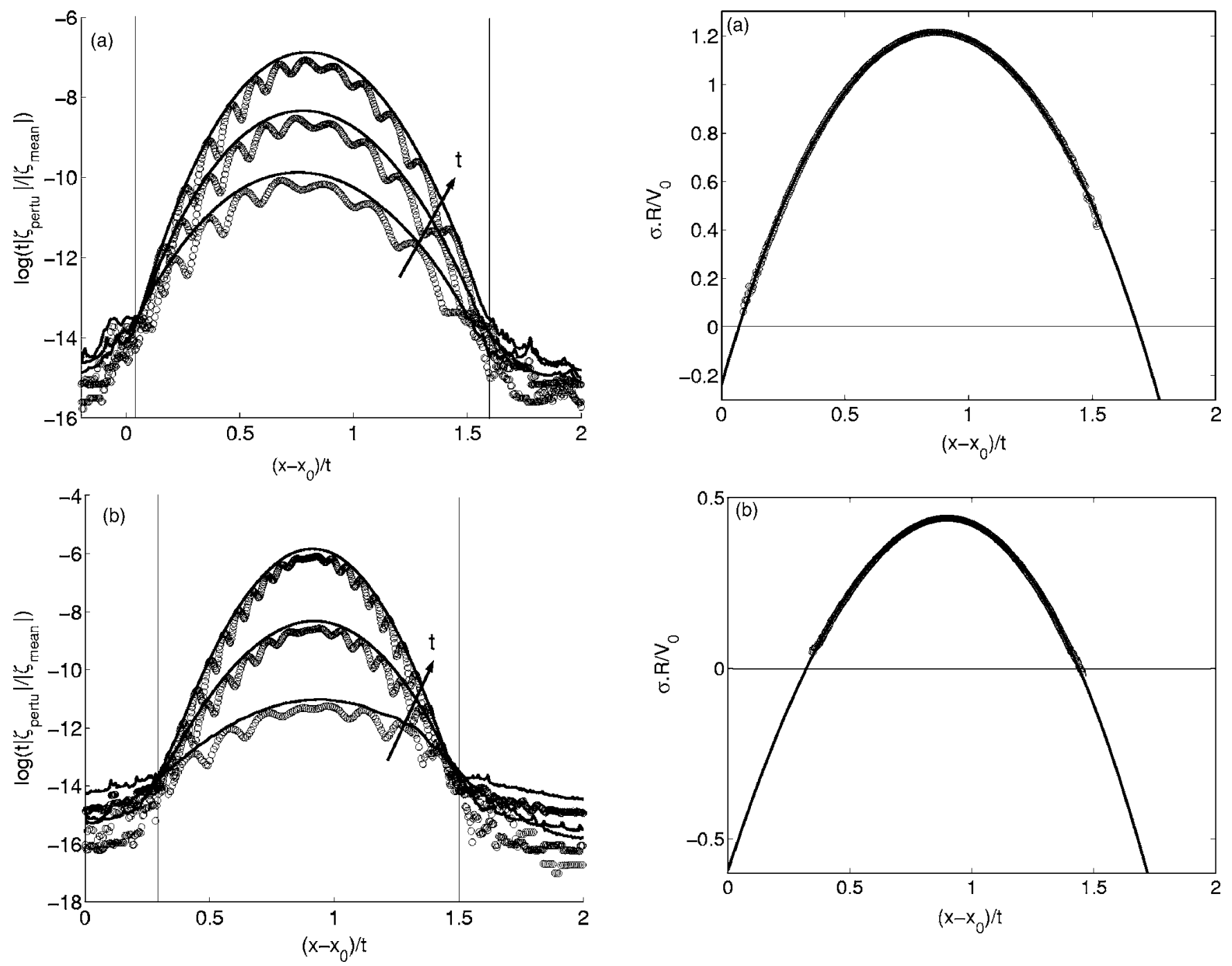

FIG. 8. Spanwise-integrated enstrophy of the wave packet (circles) and its envelope (solid line at each time) as a function of group velocity in a quasigeostrophic regime, i.e., $\lambda=0.1$, at $T=2 \tau$ with $\tau=D / V_{0}, T=2.75 \tau$, and $T=3.5 \tau$ (a) and a frontal regime, i.e., $\lambda=1.0$, at $T=2.9 \tau, T=5.9 \tau$, and $T=8.9 \tau$.

We plot this estimated spatio-temporal growth rate as a function of the group velocity $v_{g}$ for the symmetric wake in a quasigeostrophic regime and the asymmetrical wake in a frontal regime (Fig. 9, dots). The curve is incomplete since the background noise grows as well as the wave packet due to the fact that the code is not written in perturbation (but computes the evolution of the perturbation superposed to the base flow). This limits the accuracy since the amplitude close to the edges is of the order of the round off error. To better estimate the growth rate near the trailing and leading edges, we fit the curve using a fourth-order polynomial (solid line of Fig. 9). In a quasigeostrophic regime, the maximum growth rate obtained in the temporal analysis of Sec. III, $\sigma_{\max }=1.20$, is very close to $\sigma=1.21$ calculated in the spatiotemporal analysis. In a frontal regime, the difference is more important: $\sigma_{\max }=0.57$ in the linear temporal analysis whereas it is only $\sigma=0.44$ for the spatio-temporal analysis. We can argue that the perturbation evolves more slowly in a frontal regime than in a quasigeostrophic regime and the time inter-

FIG. 9. Growth rate of wave packet as a function of the group velocity for the symmetrical wake profile [Fig. 3(a)] in a quasigeostrophic regime (a) and for the asymmetrical wake profile [Fig. 3(b)] in a frontal regime (b).

val $t_{2}-t_{1}$ used to calculate the growth rate from spanwise integrated enstrophy [Eq. (6)] is taken later, $t_{1}=3 \tau$ in a frontal regime and only $t_{1}=2 \tau$ in the quasigeostrophic case. As the basic state is not maintained, the profile is more diffused in a frontal regime at the time we measured $\sigma$.

The sign of the trailing edge velocity $v_{g t}$, where $\sigma\left(v_{g t}\right)$ $=0$, determines the nature of the instability. If $v_{g t}>0$, the instability is convective. If $v_{g t}<0$, the instability is absolute. It appears that the instability of the wake profile is convective for both the quasigeostrophic and the frontal cases. The convective nature of the instability may be quantified more precisely by the value of the trailing edge group velocity. According to Fig. 9, the wave packet leaves from the cylinder with a velocity of $0.34 V_{0}$ in a frontal regime, whereas in a quasigeostrophic regime it goes much more slowly at $0.14 V_{0}$. Hence, the wake profile just behind the cylinder is much more convective in a frontal regime than in a quasigeostrophic regime.

The weakly convective nature of the instability in a quasigeostrophic regime was expected since the stability of the flow in this regime should be close to the 2D incompress- 
ible dynamics that is fully recovered when Ro is asymptotically small. In that incompressible case, Pier $^{25}$ showed that the wake flow is locally convectively unstable just behind the cylinder. This convectively unstable zone ends in the streamwise direction at $x=0.2 \mathrm{D}$ approximately while further down an absolutely unstable zone is present. As predicted both by linear or nonlinear WKB theory, ${ }^{9,20,21,26}$ the absolute unstable region is believed to trigger a global instability with a frequency determined by the absolute frequency, predicting a Strouhal number around 0.2 at moderate Reynolds number, quite far from the most unstable temporal mode prediction. These features are very similar to those found presently. Therefore, we conjecture that the behavior of the wake in the quasigeostrophic case is also due to a global instability associated with a change in nature of the local instability from convective to absolute.

The dynamics is different in the frontal regime since the flow is nearly steady and parallel several diameters downstream from the cylinder (Fig. 1) and therefore the convective instability found just behind the cylinder applies to the whole near-wake region. For such a strongly convective flow in a large region behind the cylinder, no global mode due to the presence of an absolute instability region is unstable and the late development of the perturbations observed in the experiment may be due to convective amplification of perturbation. The present observation of a preferred frequency predicted by the leading temporal instability mode agrees entirely with the theoretical predictions for everywhere convectively unstable flow.

These results show that the nature of the wake flow instability changes from absolute in the quasigeostrophic case to convective in a frontal case.

\section{CONCLUSION}

This study was motivated by the unusual wake pattern observed experimentally in a frontal regime. ${ }^{8}$ Indeed, for large geopotential fluctuations (i.e., surface deformations), only anticyclones emerge in the far wake. In order to better understand which mechanisms control this strong cycloneanticyclone asymmetry, this paper is devoted to parallel wake flow stability analysis. We studied the linear and the nonlinear evolution of two particular parallel wakes associated with symmetric and asymmetric velocity profiles measured just behind the cylinder in two different laboratory experiments. We therefore restricted this study to a local stability analysis of the wake. However, we found that this first step already gives useful results to understand both the cyclone-anticyclone asymmetry of the vortex street and the transition in Strouhal number selection and organization of the wake from the quasigeostrophic to the frontal regime.

In the quasigeostrophic regime, only the asymmetric wake flow that has a stronger anticyclonic shear induces an asymmetry in the vortex street.

For the frontal regime, we found that the linear stability of both a symmetric and an asymmetric wake flows induces a selective destabilization of the anticyclonic vorticity region. Indeed, the most unstable modes is, for both profiles, fully localized in the anticyclonic shear region. Therefore, in this regime, already from the linear stage of the instability, a significant cyclone-anticyclone asymmetry appears even if the parallel wake flow is symmetrical.

From fully nonlinear simulations, we learn that in the frontal regime cyclonic vortices are stretched and strongly deformed in comparison with the anticyclonic vortices, which remain robust and more circular. Nonlinear interactions between coherent structures of opposite sign seem to enhance the cyclone-anticyclone asymmetry. More surprisingly, we recover with this local nonlinear computation the vortex street pattern and the Strouhal number observed experimentally in the frontal regime. The asymmetry in the vortex street pattern is characteristic of the frontal regime and does not occur in the quasigeostrophic regime where both cyclones and anticyclones have about the same size and shape even when the base velocity profile is asymmetric. Note that for the incompressible $2 \mathrm{D}$ wake ${ }^{25}$ or for the present quasigeostrophic wake, since the perturbations are selfsustained due to the presence of an absolutely unstable region downstream of the cylinder, a local temporal analysis predicts an estimate of the Strouhal number three to four times too large. Hence, this strongly suggests a change in the nature of the wake instability when the geopotential fluctuations become large enough.

The change in the nature of the instability is confirmed by the spatio-temporal analysis of the asymmetric wake in a frontal regime. In this case, we found that the wake flow measured behind the cylinder is strongly convectively unstable. Since the near wake flow is nearly parallel in the frontal regime, this unstable wake would behave like a noise amplifier, the entire flow being convectively unstable. A small wave-packet perturbation is amplified but propagates downstream in the flow and does not contaminate the entire flow, as the case for the classical 2D von Karman wake. Hence, this study shows that when the frontal regime is reached, the nature of geophysical wake instability in rotating shallow-water flows undergoes a transition which impacts both the Strouhal number selection and the development of the wake.

\section{ACKNOWLEDGMENTS}

We are very thankful to Laurette Tuckerman for improving our paper. The computation has been performed on the NEC-SX5 of IDRIS in Orsay, France, under the contract number 51667.

\footnotetext{
${ }^{1}$ A. Stegner, T. Pichon, and M. Beunier, "Elliptical-inertial instability of rotating Karman streets," Phys. Fluids 17, 066602 (2005).

${ }^{2}$ L. M. Polvani, J. C. McWilliams, M. A. Spall, and R. Ford, "The coherent structures of shallow-water turbulence: Deformation-radius effects, cyclone/anticyclone asymmetry and gravity-wave generation," Chaos $\mathbf{4}$, 177 (1994).

${ }^{3}$ L. P. Graves, J. C. McWilliams, and M. T. Montgomery, "Vortex evolution due to straining: A mechanism for dominance of strong, interior anticyclones," Geophys. Astrophys. Fluid Dyn. 100, 151 (2006).

${ }^{4}$ A. C. Kuo and L. M. Polvani, "Nonlinear geostrophic adjustment, cyclone/ anticyclone asymmetry and potential vorticity rearrangement," Phys. Fluids 12, 1087 (2000).

${ }^{5}$ J. M. Baey and X. Carton, "Vortex multipoles in two-layer rotating shallow-water flows," J. Fluid Mech. 460, 151 (2002).
} 
${ }^{6}$ A. Stegner and D. G. Dritschel, "A numerical investigation of the stability of isolated shallow water vortices," J. Phys. Oceanogr. 30, 2562 (2000).

${ }^{7}$ M. Arai and T. Yamagata, "Asymmetric evolution of eddies in rotating shallow water," Chaos 4, 163 (1994).

${ }^{8}$ G. Perret, A. Stegner, M. Farge, and T. Pichon, "Cyclone-anticyclone asymmetry of large-scale wakes in the laboratory," Phys. Fluids $\mathbf{1 8}$ 036603 (2006).

${ }^{9}$ J. M. Chomaz, P. Huerre, and L. G. Redekopp, "Bifurcations to local and global modes in spatially developing flows," Phys. Rev. Lett. 60, 25 (1988).

${ }^{10}$ G. Triantafyllou, M. Triantafyllou, and C. Chryssostomidis, "On the formation of vortex streets behind stationary cylinders," J. Fluid Mech. 170, 461 (1986).

${ }^{11} \mathrm{P}$. A. Monkewitz, "The absolute and convective nature of instability in two-dimensional wakes at low Reynolds numbers," Phys. Fluids 31, 999 (1988).

${ }^{12}$ W. Koch, "Local instability characteristics and frequency determination of self-excited wake flows," J. Sound Vib. 99, 53 (1985).

${ }^{13}$ M. Provensal, C. Mathis, and L. Boyer, "Bénard-von Karman instability: Transient and forced regimes," J. Fluid Mech. 182, 1 (1987).

${ }^{14}$ G. E. M. Karniadakis and G. S. Triantafyllou, "Frequency selection and asymptotic states in laminar wakes," J. Fluid Mech. 199, 411 (1989).

${ }^{15}$ D. A. Hammond and L. G. Redekopp, "Global dynamics of symmetric and asymmetric wakes," J. Fluid Mech. 331, 231 (1997).

${ }^{16}$ P. Ripa, "General stability conditions for zonal flows in a one-layer model on the $\beta$-plane or the sphere," J. Fluid Mech. 126, 463 (1983).
${ }^{17}$ B. Cushman-Roisin, "Frontal geostrophic dynamics," J. Phys. Oceanogr. 16, 132 (1986).

${ }^{18}$ B. Cushman-Roisin and B. Tang, "Geostrophic turbulence and emergence of eddies beyond the radius of deformation," J. Phys. Oceanogr. 20, 97 (1990).

${ }^{19}$ E. Anderson, Z. Bai, C. Bischof, S. Blackford, J. Demmel, J. Dongarra, J. Du Croz, A. Greenbaum, S. Hammarling, A. McKenney, and D. Sorensen, LAPACK Users' Guide, 3rd ed. (Society for Industrial and Applied Mathematics, Philadelphia, PA, 1999).

${ }^{20}$ J. M. Chomaz, "Fully nonlinear dynamics of parallel wakes," J. Fluid Mech. 495, 57 (2003)

${ }^{21}$ P. Huerre and P. A. Monkewitz, "Local and global instabilities in spatially developing flows," Annu. Rev. Fluid Mech. 22, 473 (1990).

${ }^{22}$ F. J. Poulin and G. R. Flierl, "The nonlinear evolution of barotropically unstable jets," J. Phys. Oceanogr. 33, 2173 (2003).

${ }^{23}$ A. Bers, "Space-time evolution of plasma instabilities-Absolute and convective," in Handbook of Plasma Physics, edited by M. Rosenbluth and R. Sagdeev (North-Holland, Amsterdam, 1983), Vol. 1, p. 451.

${ }^{24}$ I. Delbende, J. M. Chomaz, and P. Huerre, "Absolute/convective instabilities in the Batchelor vortex: A numerical study of the linear impulse response," J. Fluid Mech. 355, 229 (1998)

${ }^{25} \mathrm{~B}$. Pier, "On the frequency selection of finite-amplitude vortex shedding in the cylinder wake," J. Fluid Mech. 458, 407 (2002).

${ }^{26}$ J. M. Chomaz, "Global instabilities in spatially developing flows: Nonnormality and nonlinearity," Annu. Rev. Fluid Mech. 37, 357 (2005). 